\title{
Very strong correlation between dominant negative activities of mutant thyroid hormone receptors and their binding avidity for corepressor SMRT
}

\author{
A Matsushita, H Misawa, S Andoh, H Natsume, K Nishiyama, \\ S Sasaki and $\mathbf{H}$ Nakamura \\ Second Division, Department of Internal Medicine, Hamamatsu University School of Medicine, 3600 Handacho, Hamamatsu, Japan \\ (Requests for offprints should be addressed to H Nakamura; Email: hirotosh@hama-med.ac.jp)
}

\begin{abstract}
The syndrome of resistance to thyroid hormone (RTH) is an inherited disorder involving a mutation of the thyroid hormone receptor (TR) gene. Mutant (m) TR inhibits wild-type (wt) TR functions in a dominant negative manner, and this dominant negative effect (DNE) is a crucial factor in RTH pathogenesis. The molecular mechanism of the DNE is still unclear, although several possibilities (including competition between wt- and mTRs at the $\mathrm{T}_{3}$ response element (TRE), sequestration of TR-associated protein(s) and titration out of functional TR) have been considered. Here we report that the DNE of mTRs is strongly correlated with their binding avidity for the retinoid X receptor (RXR), and especially for corepressor SMRT (silencing mediator for retinoid and thyroid hormone receptor), but not for the nuclear receptor corepressor, NCoR. The DNE of six natural TRs and four artificially constructed mTRs was assayed using a TR reporter gene containing TRE-DR4 (DR = direct repeat), TRE-pal (pal=palindrome) or TRE-lap $($ lap =inverted palindrome) in CV1 cells treated with $10 \mathrm{nM} \mathrm{T}_{3}$. Of the mTRs examined, F451X (with a carboxy-terminal 11amino-acid truncation) identified in a patient with RTH exhibited the strongest DNE on all TREs. The binding affinities between mTRs and corepressors SMRT or
\end{abstract}

NCoR were quantified using a two-hybrid interference assay system consisting of VP16-TR(LBD) (LBD=ligand binding domain) and Gal4(DBD)-SMRT (DBD=DNA binding domain), or Gal4(DBD)-NCoR respectively, together with the Gal4 reporter gene. In this assay, VP16-TR(LBD) and Gal4(DBD)-SMRT (or Gal4 (DBD)-NCoR) interact with each other and trans-activate the Gal4 reporter gene. When an equal amount of mTR is coexpressed, it reduces the transcriptional activity of the reporter gene, depending on its binding avidity for a corepressor. A very strong correlation was observed between the SMRT-binding activity and the potency of the DNE among six natural mTRs and also among all mTRs, including four artificially constructed ones. The relationship between NCoR and DNE, however, was not significant. When we assayed the binding avidity of mTRs for RXR by using a two-hybrid assay system consisting of Gal4(DBD)-RXR(LBD) and VP16-TR(LBD), a significant correlation between DNE and binding avidity for the RXR was also observed. These results suggest that a corepressor plays an important role in DNE pathogenesis.

Journal of Endocrinology (2000) 167, 493-503

\section{Introduction}

The syndrome of resistance to thyroid hormone (RTH) is an inherited disorder involving target-tissue hyporesponsiveness to thyroid hormone (TR). Since the first report by Refetoff et al. (1967), many patients with RTH have been described. In most of them, nucleotide substitutions in one of their TR $\beta$ alleles have been identified. Because a patient with RTH has one normal TR $\beta$ allele as well as two normal TR $\alpha$ alleles, the abnormal $\operatorname{TR} \beta$ allele is thought to inhibit the functioning of the normal TR alleles. The parents of the patient first reported by Refetoff et al. (1967) were phenotypically normal in spite of their loss of one TR $\beta$ allele (Takeda et al. 1992). This suggests that a decrease in the amount of TR itself is not sufficient to induce RTH, and that the presence of an abnormal $\operatorname{TR} \beta$ allele is crucial.

The molecular mechanism by which mutant $(\mathrm{m})$ TR inhibits the functions of wild-type (wt) TR in a dominant negative manner is still unclear. The DNA-binding properties of mTRs and their capacity to dimerize activity with the retinoid X receptor (RXR) are known to be important in dominant negative inhibition (Meier et al. 1993). It has been reported that artificially constructed $\operatorname{mTR} \beta$ 
alleles with amino acid substitutions in the DNA-binding domains and dimerization regions do not exhibit dominant negative effects (DNEs) (Nagaya et al. 1996). One hypothesis is that mTR forms a functionally inactive heterodimeric complex with the RXR and binds to the TRE, thereby competing with the normal TR/RXR for a target DNA site. However, if competition between $\mathrm{mTR} / \mathrm{RXR}$ and wtTR/RXR on a TRE is the only causative mechanism for the DNE, the strength of the DNE should be completely dependent on the affinity of binding to the TRE. No data have been reported showing that mTRs with stronger DNEs have stronger TREbinding activities, or that the binding affinities for the TRE among various $\mathrm{mTR} / \mathrm{RXR}$ heterodimers are strongly correlated with the potencies of their DNEs. It is therefore thought that some additional causative factors are likely to be involved in RTH pathogenesis.

In recent years, many transcriptional co-activators, including those of the SRC family and others, have been identified (McKenna et al. 1999). Mutant TR $\beta$ may sequester some of these important transcriptional coactivators. In addition to co-activators, corepressors such as SMRT (silencing mediator for retinoid and thyroid hormone receptor) and NCoR (nuclear receptor corepressor) have also been shown to mediate the silencing activity of unliganded TR and the retinoic acid receptor (RAR) (Chen \& Evans 1995, Horlein et al. 1995). Recent reports suggest that binding of some mTR $\beta$ s with corepressors is required for the DNE (Yoh et al. 1997, Nagaya et al. 1998, Tagami \& Jameson 1998). Here we report that the strength of the DNE among natural mTRs is strongly correlated with their binding avidity for the corepressor SMRT, suggesting that SMRT plays an important role in DNE pathogenesis.

\section{Materials and Methods}

\section{Plasmids}

The following plasmids were used in the experiments: pCMX expression vectors containing wt human TR $\beta 1$ (pCMX-hTR $\beta 1$ ), the natural mutants TR $\beta 1 \mathrm{~F} 451 \mathrm{X}$ (Miyoshi et al. 1995), R338W (Sasaki et al. 1993), K443E (Sasaki et al. 1992), G345R (Sakurai et al. 1989), E449X (Miyoshi et al. 1998) and L456fs (Bhat et al. 1995), and the artificially constructed mutants TR $\beta 1$ L428R (Nagaya \& Jameson 1993), R338W/K443E (Andoh et al. 1996), R338W/F451X (Andoh et al. 1996) and G345R/K443E (Andoh et al. 1996). Their $T_{3}$-binding, DNA-binding and transcriptional activities had been reported previously. All of the mTRs had severely impaired $\mathrm{T}_{3}$-binding activities (Nagaya \& Jameson 1993, Andoh et al. 1996, Miyoshi et al. 1998), except for R338W, which showed a tendency towards slightly, but not significantly, higher $\mathrm{T}_{3}$ binding compared with other mutant TRs (Andoh et al. 1996). The mammalian two-hybrid interference assays involved the use of $\mathrm{pCMX}$ expression vectors containing the fusion proteins Gal4(DBD)-RXR $\alpha(\mathrm{LBD})$, Gal4(DBD)-SMRT, and Gal4(DBD)-NCoR, in which the carboxy-terminus of the DNA-binding domain (DBD) of yeast nuclear transcription factor Gal4 was fused to the ligand-binding domain (LBD) of human RXR $\alpha$, the interacting domain of human corepressors SMRT or NCoR respectively and VP16-TR(LBD), in which the activation domain of herpes simplex virus transcriptional factor VP16 was linked to the amino-terminus of the LBD of human TR $\beta 1$. As reporter genes, a chloramphenicol acetyl transferase (CAT) reporter gene containing TRE-DR4, two copies of a palindrome (pal2) and an inverted palindrome (lap), and a luciferase gene containing three copies of Gal4 response element (Galpx3-tk-Luc) were used. The nucleotide sequences of TREs used in this study have been described elsewhere (Nishiyama et al. 1998). The structures of the mTRs used in this study are shown in Fig. 1.

Cell culture, transient transfection and measurement of DNEs of $m$ TRs

CV1 cells were grown in Dulbecco's modified Eagle's medium (DMEM) containing 10\% fetal calf serum (FCS), penicillin $\mathrm{G}(100 \mathrm{U} / \mathrm{ml})$ and streptomycin $(100 \mu \mathrm{g} / \mathrm{ml})$. The cells were trypsinized and plated in $60-\mathrm{mm}$ dishes $24 \mathrm{~h}$ prior to transient transfection using calcium phosphate. Cells at a density of $10^{6}$ cells/plate were transfected with $0.18 \mu \mathrm{g}$ wt- and/or mTR $\beta 1$ expression vector along with $1.8 \mu \mathrm{g}$ CAT reporter gene, $3.6 \mu \mathrm{g} \beta$-galactosidase expression vector pCH111 (a modified version of pCH110; Pharmacia LKB Biotechnology, Piscataway, NJ, USA) and pBlueScript II-SK(+) (Stratagene, La Jolla, CA, USA) as carrier DNA (a total of $7 \cdot 2 \mu \mathrm{g}$ DNA per dish). After the cells had been exposed to the calcium phosphate/DNA precipitate for $20 \mathrm{~h}$, the medium was replaced with fresh DMEM containing 10\% FCS depleted of thyroid hormone (Samuels et al. 1979), supplemented with $10 \mathrm{nM} \mathrm{T}$. After incubation for an additional $24 \mathrm{~h}$, the cells were harvested and the CAT activity was measured as described previously (Nordeen et al. 1987). The transfection efficiency was normalized using the $\beta$-galactosidase assay.

The CAT activity was measured in $10 \mathrm{nM} \mathrm{T}_{3}$-treated CV1 cells expressing wtTR only or expressing equal amounts of wt- and mTRs. The dominant negative effect of mTR was calculated as the \% of the CAT activity driven by wtTR. Our previous data (Andoh et al. 1996) and current study (data not shown) demonstrated that the DNEs of many mTRs were maximal at $10 \mathrm{nM} \mathrm{T}_{3}$ when various concentrations $(1 \mathrm{nM}$ to $1 \mu \mathrm{M})$ of $\mathrm{T}_{3}$ were tested. The dominant inhibitory potency of mTR was calculated as the ratio between the CAT activities in cells expressing wtTR and those in cells expressing both wt- and mTR. 


\begin{tabular}{|c|c|c|c|c|}
\hline & & & & \\
\hline TRß1-Wild & $A / B$ & C & D & $E / F$ \\
\hline
\end{tabular}
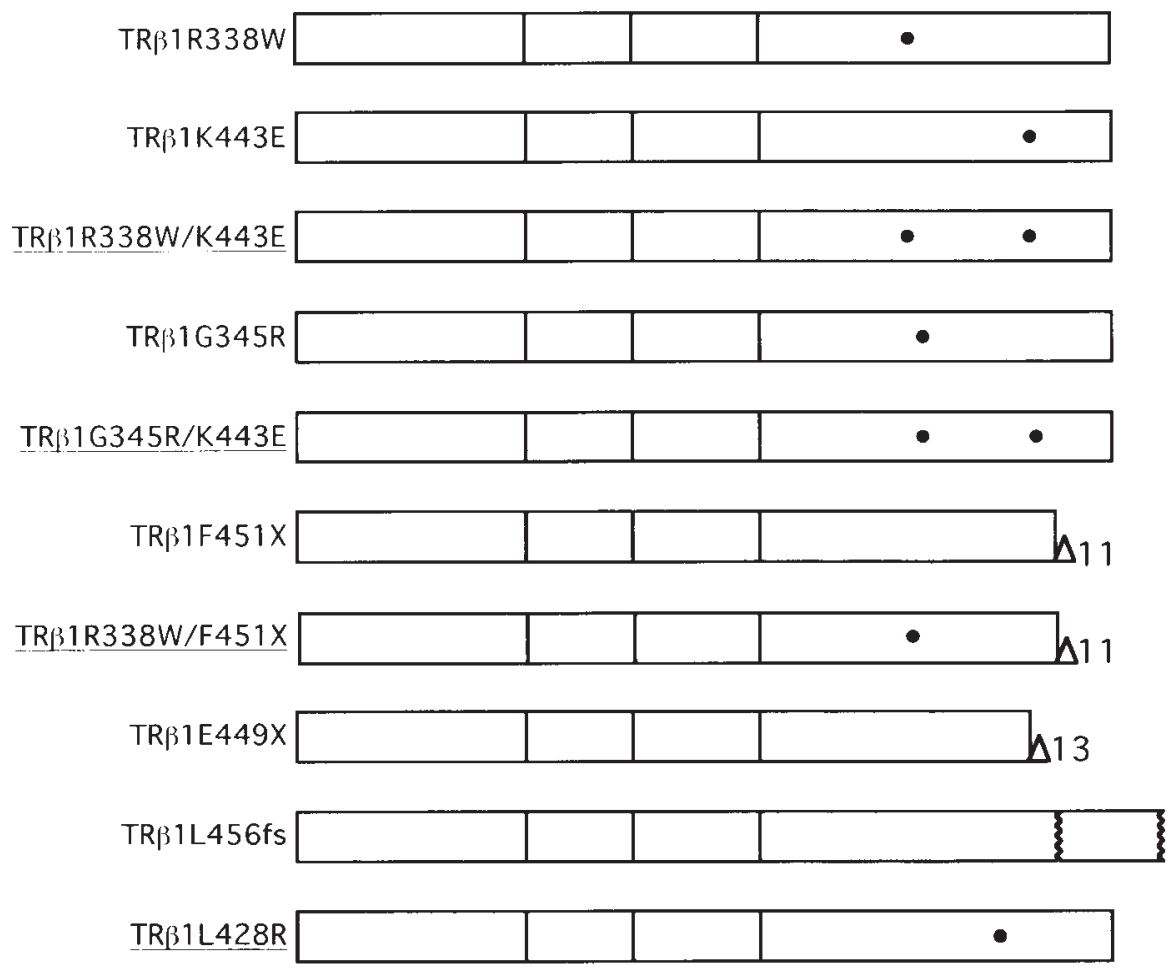

Figure 1 Structures of the mTRs used in the study. Artificially constructed mTRs are underlined. $\Delta 11$ and $\Delta 13$ indicate the carboxy-terminal 11 - and 13-amino-acid deletions respectively.

- Amino acid substitutions; fs, frame shift.

\section{Mammalian two-hybrid interference assay}

The expression plasmids for Gal4(DBD)-RXR(LBD) and VP16-TR(LBD) were transfected into CV1 cells along with the Gal4 reporter plasmid (Galpx3-tk-Luc). To detect interference activities of wt- and mTRs, pCMX encoding wtTR $\beta 1$ or mTR was cotransfected into the cells. The VP16-driven luciferase activity of the Gal4 reporter gene was taken as 100\% when empty expression vector pCMX was transfected (the control activity), and was expressed as a percentage of the control activity when wt- and mTR were coexpressed. Since coexpressed TR interferes with the Gal4(DBD)-RXR(LBD) and VP16$\mathrm{TR}(\mathrm{LBD})$ interaction by binding to the RXR(LBD), the decrease in the luciferase activity represents the RXRbinding activity of the mTR. The potency of the binding mTR to the RXR was calculated as the ratio between the control luciferase activity and the activity in cells coexpressing the mTR. Similarly, the binding activities for MTR and corepressors SMRT and NCoR were assayed in the cells expressed with VP16-TR(LBD) and Gal4(DBD)-SMRT or -NCoR respectively, together with the Gal4 reporter gene. The potency of binding of $\mathrm{mTR}$ to the corepressor was calculated in a manner similar to that used to calculate the RXR-binding potency.

\section{Statistical analysis}

The statistical significances of differences in the CAT and luciferase activities were determined by using analysis of variance (ANOVA) and Fisher's Protected Least Significant Difference test, with STATVIEW 4.0 (Abacus Concepts, Berkeley, CA, USA).

\section{Results}

Dominant negative activities of mutant TRs

Each mTR was expressed in CV1 cells together with an equal amount of wtTR, and the transcriptional activity was measured using three different reporter genes containing TRE-DR4, -pal2 or -lap. The dominant negative activities of mTRs against wtTR function were thus 

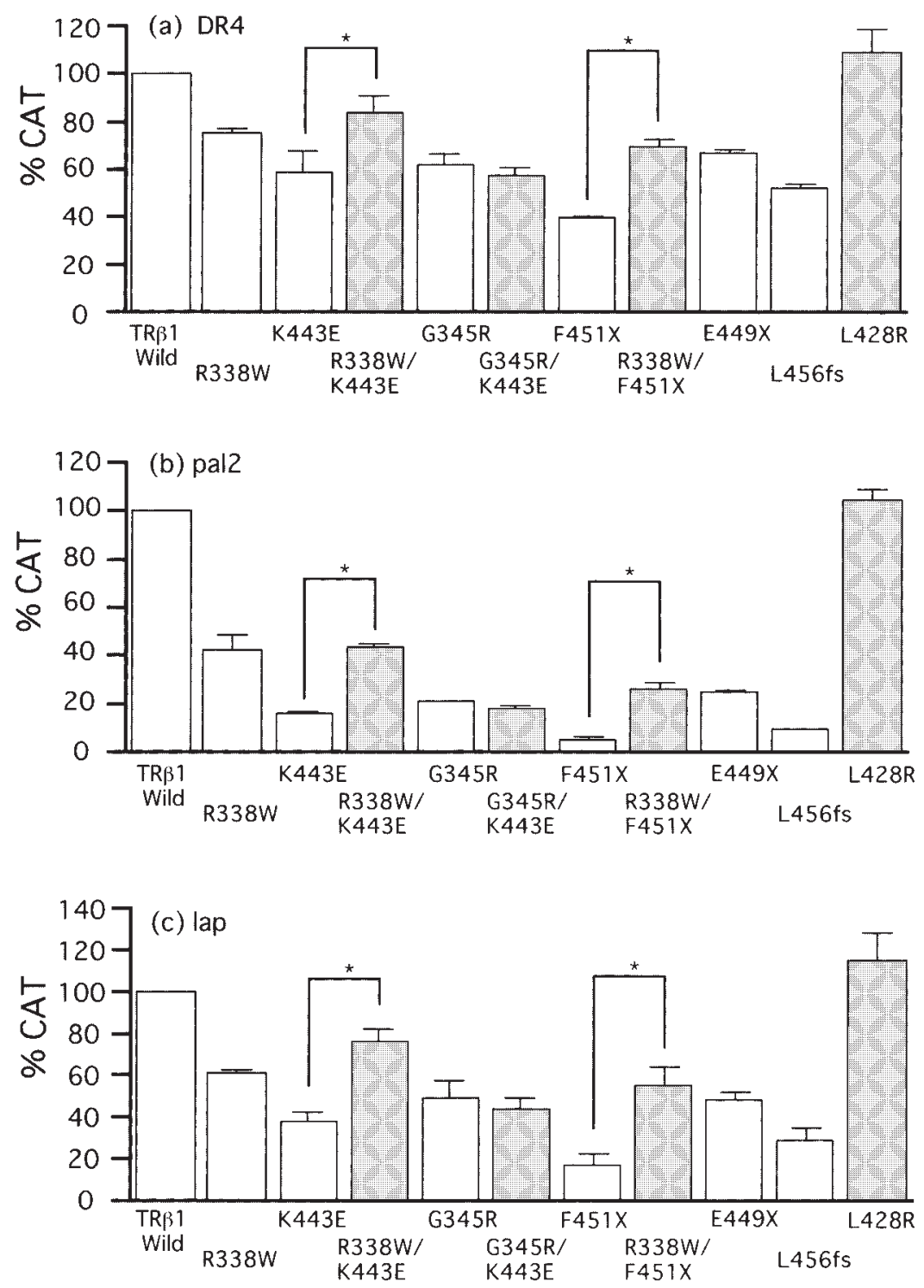

Figure 2 Dominant negative activities of mutant TRs. Expression vectors for wt- and mTR $\beta 1 \mathrm{~s}$ were transfected into CV1 cells and the transcriptional activities were assayed using TRE-DR4 (a), TRE-pal2 (b) and TRE-lap (c) in the presence of $10 \mathrm{nM} \mathrm{T}$. The CAT activity of wt TR $\beta 1$ is expressed as $100 \%$. Artificially constructed mTRs are represented by shaded bars. Each result represents the mean \pm S.D. of four experiments $\left({ }^{*} P<0 \cdot 05\right)$.

obtained (Fig. 2). Of ten mTRs, F451X exhibited the strongest inhibition against the wtTR transcriptional activity on all three TREs. On the other hand, L428R, which has impaired dimerization capacity (Nagaya \& Jameson 1993), did not show any dominant negative inhibition of the wtTR function. As previously reported (Andoh et al. 1996), introduction of the R338W mutation, but not of the G345R mutation, weakened the dominant negative activity of K443E.
Binding avidity for SMRT of mutant TR measured by mammalian two-hybrid interference assay

The binding avidity of each mutant TR for corepressor SMRT was measured in CV1 cells by using a mammalian two-hybrid interference assay consisting of VP16$\mathrm{TR}(\mathrm{LBD})$ and Gal4(DBD) fused with the interacting domain of SMRT (Gal4-SMRT). When expressed in the cells, TR(LBD) and SMRT interacted with each other 

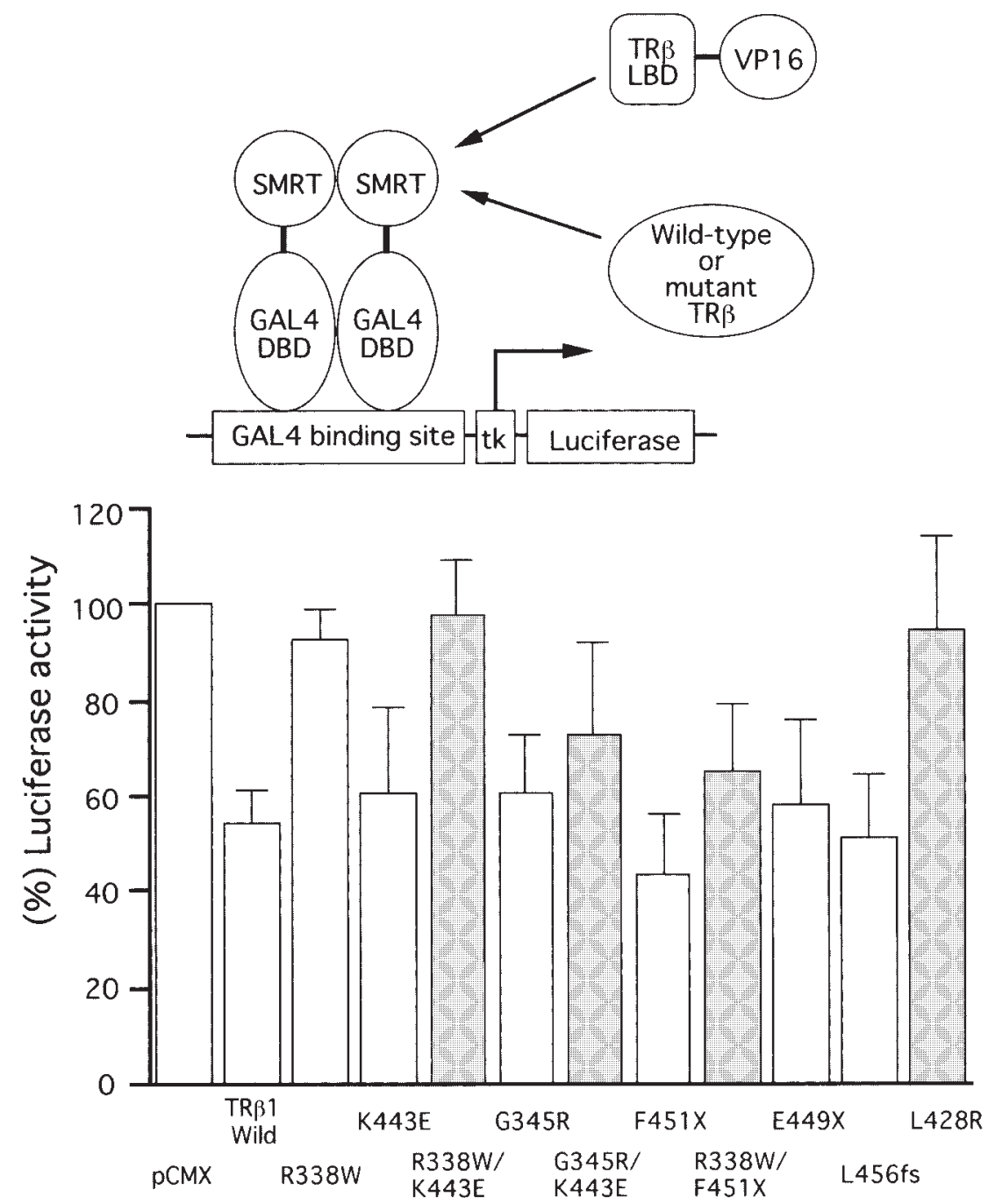

Figure 3 The binding avidity of mTR for SMRT, measured using a mammalian two-hybrid interference assay system. Expression vectors for Gal4(DBD)-SMRT and VP16-TR(LBD) were transfected into CV1 cells together with the pCMX empty vector or each mTR vector. The luciferase activities were measured using a Gal4 reporter gene. The transcriptional activity in the cells with only pCMX expression (the control activity) is shown as $100 \%$ and that with mTR expression is calculated as a percentage of the control activity. Artificially constructed mTRs are represented by shaded bars. The results are the means \pm S.D. of four experiments.

and the Gal4 reporter gene was strongly trans-activated. Coexpression of wt- or mTR reduced the luciferase activity of the reporter gene by interfering with the TR(LBD) and SMRT interaction. The decrease in the luciferase activity was dependent on the interference potency of mTR. This two-hybrid interference assay system allowed us to estimate the binding activities of $167 \mathrm{wt}-$ and mutant full-length TRs, not the LBD, to SMRT. The results are shown in Fig. 3. As expected, L428R did not interfere with Gal4(DBD)-SMRT and VP16-TR(LBD) association, whilst F451X exhibited the strongest binding, to SMRT, of all the TRs examined.
Correlation between dominant negative activity and SMRT binding avidity of mutant TRs

The DNE of each mTR was calculated for each TRE as the potency relative to wtTR, using the data shown in Fig. 2. The binding avidity for SMRT was calculated as the relative potency of interference between the Gal4(DBD)-SMRT and VP16-TR(LBD) interaction. When the DNEs of six natural mTRs identified in patients with RTH were plotted versus their SMRT binding avidity (Fig. 4, a strong correlation was observed between the two activities on all three TREs. When the 
(a) DR4

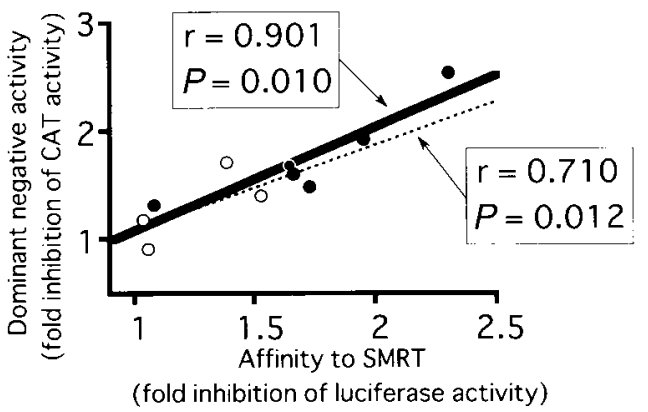

(b) pal2

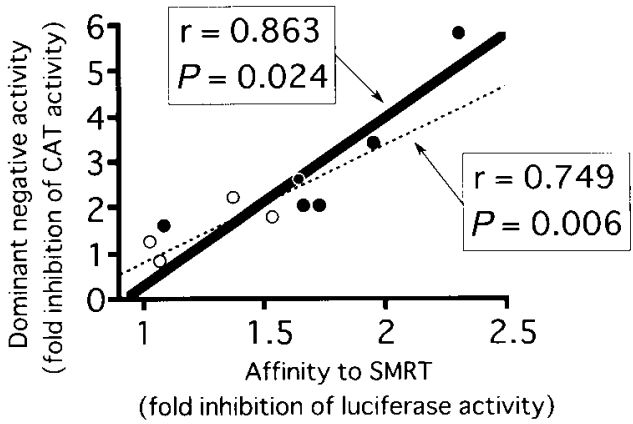

(c) lap

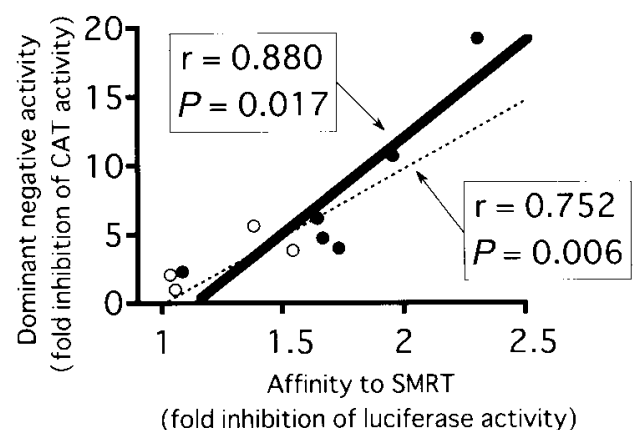

Figure 4 Correlation between the binding avidities of ten mutant TRs for SMRT and the dominant negative activities measured on TRE-DR4 (a), TRE-pal2 (b) and TRE-lap (c). The potency of the dominant negative activity was calculated as the ratio between the CAT activities with expression of wt TR and those with expression of mTR (from the data shown in Fig. 2). The binding avidity for SMRT was calculated as the ratio between the luciferase activities with expression of $\mathrm{pCMX}$ and those with expression of mTR (from the data shown in Fig. 3). Solid lines represent correlation among six natural mTRs $(-)$; dotted lines represent correlation among all mTRs, including four artificially constructed mTRs $(\bigcirc)$.

data of the four artificially constructed mTRs were included, significance of the correlation became much higher.

Binding avidity of mutant TRs for NCoR and correlation with dominant negative activity

The binding activity of mutant TRs for another corepressor, NCoR, was assayed using VP16-TR(LBD) and
Gal4(DBD) fused with the interacting domain of $\mathrm{NCoR}$ (Gal4-NCoR) expressed in CV1 cells. Expression of an empty vector (pCMX) did not interfere with the TR(LBD) and NCoR interaction, and the Gal4 reporter gene was trans-activated, whereas expression of wtand mTRs disrupted the interaction of the two-hybrid proteins, resulting in a decrease in the luciferase activity (Fig. 5). When the DNEs were plotted against the NCoR-binding activity of mTRs, no significant correlation was found between them on all three TREs among six natural mTRs or among all mTRs (Fig. 6).

\section{Binding avidity of mutant TR for RXR and correlation with dominant negative activity}

We next examined the relationship between the DNE and the RXR binding avidity of mTRs. The binding avidity of mTRs for the RXR was measured using the two-hybrid interference assay system consisting of Gal4(DBD)-RXR(LBD) and VP16-TR(LBD). The association between the LBD of wtTR and the LBD of the RXR led to activation of the Gal4 reporter gene, and mTR was coexpressed in the cells; this disrupted the association and reduced the luciferase activity of the Gal4 reporter gene, depending on the RXR binding avidity of the mTR (Fig. 7). When the binding avidity for RXR was calculated as the relative potency of interference between the Gal4(DBD)-RXR(LBD) and VP16TR(LBD) interaction and plotted against the DNE, a significant correlation was observed between them for each TRE among six natural mTRs (Fig. 8) or for all mTRs.

\section{Discussion}

Dominant negative inhibition of normal TR functions by $\operatorname{mTR} \beta$ is crucial in the pathogenesis of RTH. Several different models have been proposed for the mechanism of DNE. Most of the mTRs identified in patients with RTH have impaired $\mathrm{T}_{3}$ binding and the mTR/RXR heterodimer is transcriptionally inactive. Functionally inactive $\mathrm{mTR} / \mathrm{RXR}$ heterodimers occupy the target DNA site, thereby preventing access of a normal TR/RXR complex to the TRE. Mutant TR may dimerize with normal TR and form an inactive complex between mutant and normal TRs, resulting in titration out of the remaining functional TR. Similarly, titration out of the RXR by mTR is conceivable, but this possibility seems unlikely since expression of large amounts of the RXR does not eliminate the DNE of mTRs (Yen \& Chin 1994). Alternatively, mTR may sequester some transcriptionally important factor(s) which is/are limiting in the cells. This possibility is considered to be highly likely because, recently, many transcriptional co-activators have been identified (McKenna et al. 1999). Of course, these models 

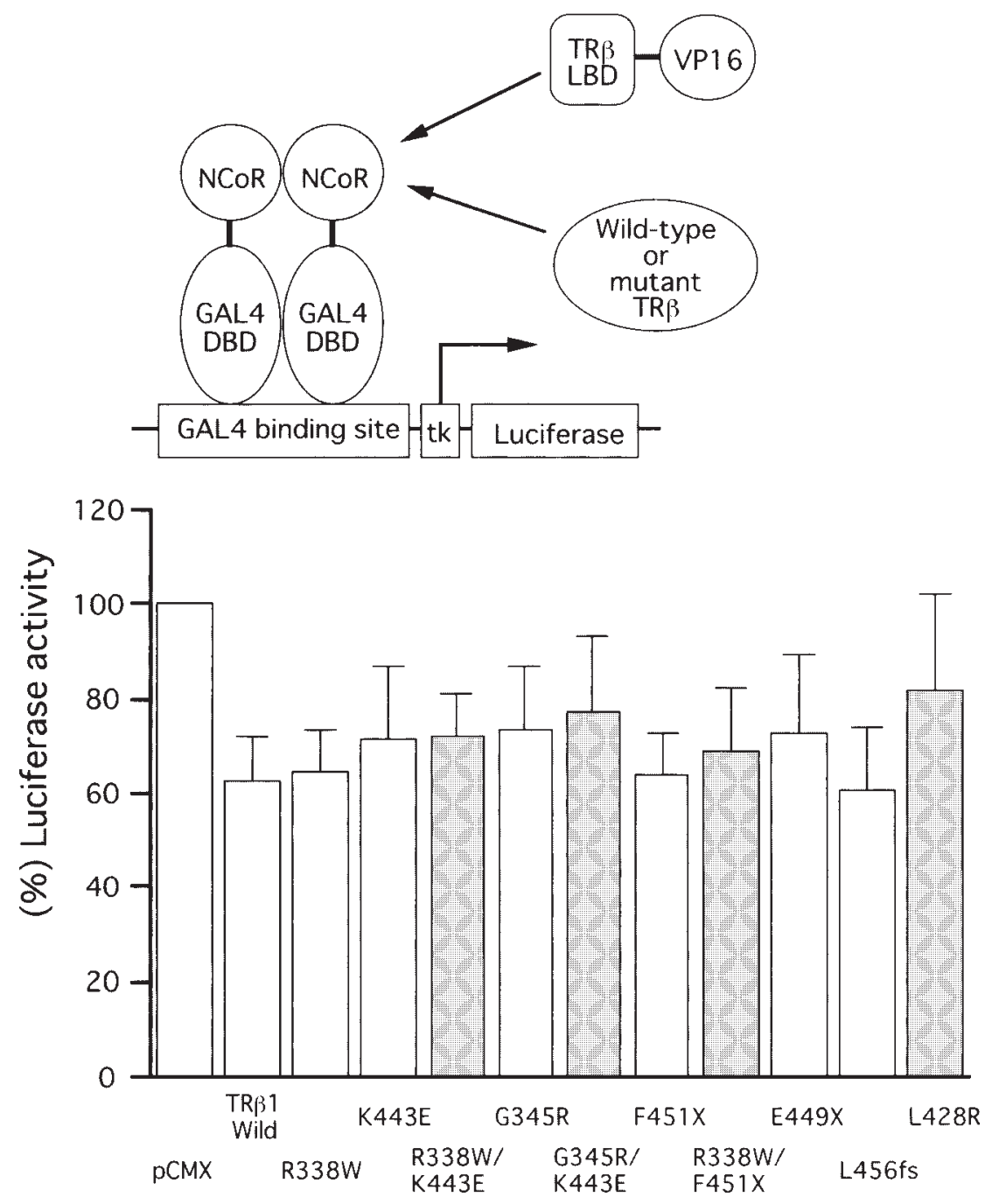

Figure 5 The binding avidity of mTR for NCoR, measured using a mammalian two-hybrid interference assay system. Expression vectors for Gal4(DBD)-NCoR and VP16-TR(LBD) were transfected into CV1 cells together with the $\mathrm{PCMX}$ empty vector or each $\mathrm{mTR}$ vector. The luciferase activities were measured using a Gal4 reporter gene. The transcriptional activity in the cells with only pCMX expression (the control activity) is shown as $100 \%$ and that with mTR expression is calculated as a percentage of the control activity. Artificially constructed mTRs are represented by shaded bars. The results represent the means \pm S.D. of four experiments.

are not mutually exclusive; mTR/RXR heterodimers may compete with the normal TR/RXR for the TRE and titrate out a transcriptional factor whilst simultaneously reducing the amount of functional TR.

Many co-activators and corepressors that are required by TR for efficient transcriptional regulation have been identified. The association of these transcriptional cofactors with DNE has recently been investigated. For example, Collingwood et al. (1997) found that a natural mTR of RTH, L454 V, had a markedly reduced ability to interact with SRC-1 and that coexpression of SRC-1 restored the transcriptional activity of mutant L454 V. They also reported that another natural mutant, T277A, exhibited an impaired transcriptional activity because of its reduced co-activator binding (Collingwood et al. 1998). Liu et al. (1998) reported data suggesting that inability to interact with co-activators such as SRC- 1 could be a determinant of the DNE of mTRs. On the other hand, Clifton-Bligh et al. (1998) reported that disproportionately impaired release of corepressors from mutant receptor $\mathrm{R} 383 \mathrm{H}$ by $\mathrm{T}_{3}$ might mediate the DNE on negatively regulated genes. Similarly, Tagami et al. (1998) found that L454S had a 
(a) DR4

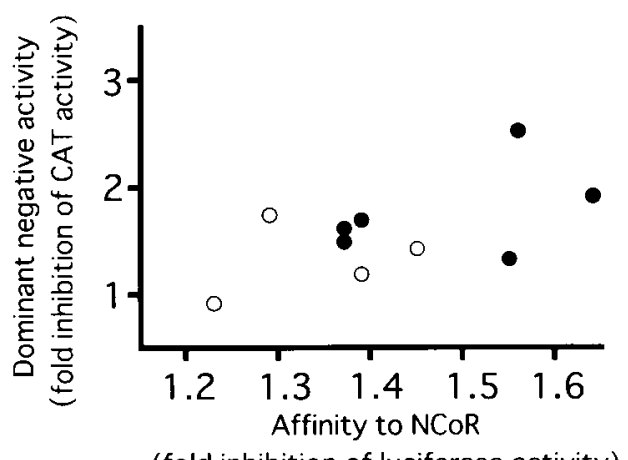

(b) pal2

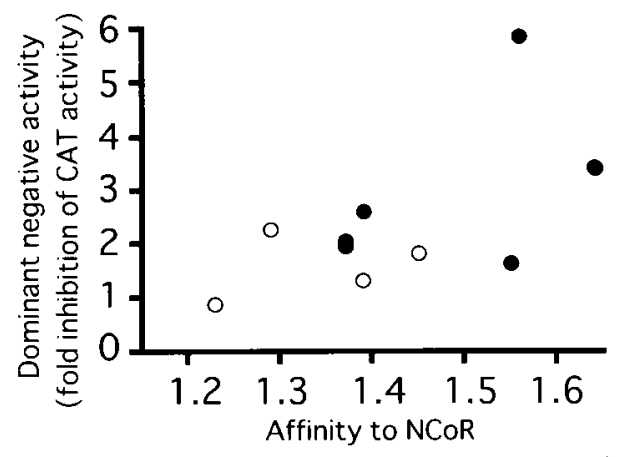

(c) lap

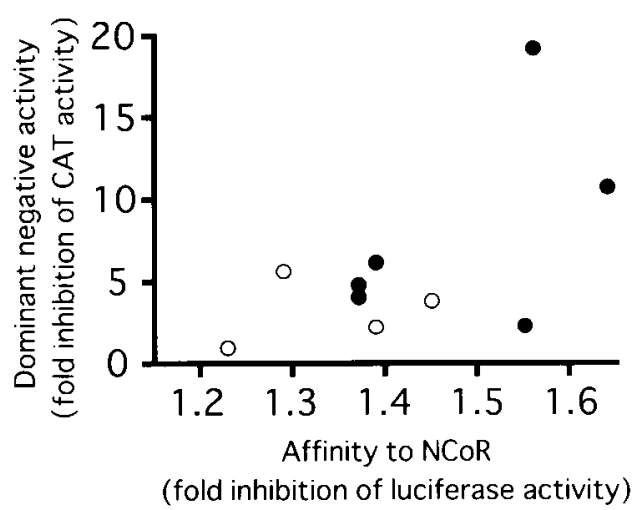

Figure 6 Correlation between the binding avidities of six natural mTRs $(\bigcirc)$ and four artificially constructed mTRs $(O)$ for NCoR and the dominant negative activities measured on TRE-DR4 (a), TRE-pal2 (b) and TRE-lap (c). The potency of the dominant negative activity was calculated as the ratio between the CAT activities with expression of wt TR and those with expression of mTR (from the data shown in Fig. 2). The binding avidity for NCoR was calculated as the ratio between the luciferase activities with expression of pCMX and those with expression of mutant TR (from the data shown in Fig. 5).

very strong interaction with corepressor $\mathrm{NCoR}$ in the absence of $\mathrm{T}_{3}$ and markedly impaired NCoR release by $\mathrm{T}_{3}$, thereby exhibiting remarkable DNE. Safer et al. (1998) found that mTRs with a mutation in the hinge region had reduced $\mathrm{T}_{3}$ binding when complexed to DNA, preventing $\mathrm{NCoR}$ dissociation from the receptors. Recent studies (e.g. on P214R) have shown that the introduction of a mutation into the hinge region abolishes or abrogates the DNE of RTH mutants (Tagami \& Jameson 1998, Nagaya et al. 1998). Although these studies of CoR-box mutations suggest that a corepressor interaction plays a critical role in the DNE, the idea that introduction of a mutation into the hinge region may change the TR structure drastically, resulting in some unexpected alterations in the ligand and co-activator association, is a possibility that cannot be completely ruled out. In the present study, we measured the binding of ten $\operatorname{mTR} \beta$ s to the RXR, the corepressor SMRT and NCoR, using a mammalian two-hybrid interference assay system in CV1 cells. We found a very strong correlation between binding avidity for corepressor SMRT and dominant negative potency among six natural mTRs. The correlation coefficients were surprisingly high on three different TREs. The affinity of binding to the RXR also showed a significant, but less remarkable, correlation with the DNE. Our data in this study lend further support to the view that a corepressor is important in DNE pathogenesis.

It is interesting that the binding avidity for another corepressor, $\mathrm{NCoR}$, did not show a significant correlation with the potency of the DNE, in spite of the fact that SMRT and NCoR are highly homologous in their structures and functions (McKenna et al. 1999). However, the two corepressors may not necessarily be identical in their functional properties. In fact, it is known that SMRT and $\mathrm{NCoR}$ exhibit differences in some aspects. RevErb, for example, interacts with NCoR but not with SMRT (Zamir et al. 1996). Similarly, the orphan receptor DAX-1 interacts with NCoR, but not with SMRT (Crawford et al. 1998). In the study of the cell-specific silencing activity of the RAR $\alpha$ reported by Baniahmad et al. (1998), the RAR $\alpha$-silencing activity was potentiated sixfold by the expression of SMRT, but not NCoR, in CV1 cells, whereas no effects were observed in L cells. These workers suggested that SMRT and NCoR have differential effects on nuclear receptors in a cell-dependent manner. SMRT and NCoR are expressed ubiquitously (Misiti et al. 1998) and it is currently unclear how they play distinct roles as corepressors. Our finding indicates that SMRT, not $\mathrm{NCoR}$, is a predominant functional corepressor in CV1 cells, at least under our assay conditions.

Since the two-hybrid assay system that we used is principally based on competitive binding interference, expression of each competitor mTR protein at an equal level in the cells is required for accurate measurement of the binding avidity for the corepressor. Although it is not easy to estimate each $\mathrm{mTR}$ protein concentration accurately, we consider that the differences in DNE and the interaction with SMRT are not due to the different mTR protein expression. First, in a separate study, we constructed wt- and mTRs tagged amino-terminally with a FLAG sequence and assessed the TR proteins expressed in 


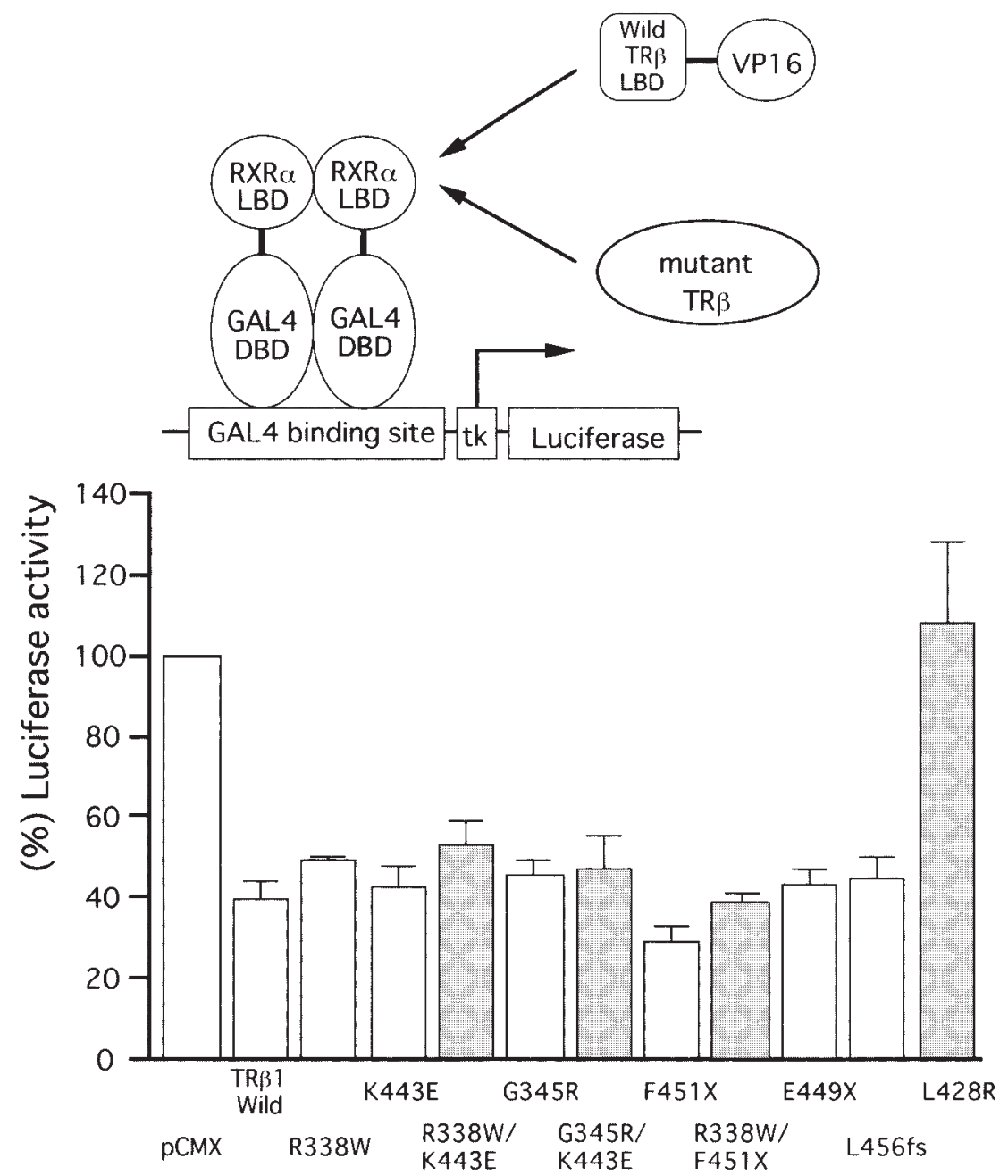

Figure 7 The RXR binding avidity of $\mathrm{mTR}$, measured using a mammalian two-hybrid interference assay system. Gal4(DBD)-RXR(LBD) and VP16-TR(LBD) with the Gal4 reporter gene in CV1 cells. The empty vector for $\mathrm{PCMX}$ or the expression vector for each $\mathrm{mTR}$ was cotransfected. Luciferase activities were measured and normalized to $\beta$-galactosidase activity. The transcriptional activity in the cells with only pCMX expression (the control activity) is shown as $100 \%$ and that with mTR expression is calculated as a percentage of the control activity. The decrease in the luciferase activity represents the binding avidity, for RXR, of each mTR. Artificially constructed mTRs are represented by shaded bars.

CV1 cells under the same experimental conditions as those used in the present study by immunoblot analysis using anti-FLAG antibody. The immunoblot analysis demonstrated no apparent differences in the densities of the FLAG-fused TR bands among wt TR $\beta 1, \beta F 451 X$ and $\beta E 449 \mathrm{X}$ and among wt TR $\alpha 1, \alpha \mathrm{F} 397 \mathrm{X}$ and $\alpha \mathrm{E} 395 \mathrm{X}$ (Nishiyama et al. 2000). Secondly, the DNE potencies of mTRs were strongly correlated with their SMRT binding avidities, but not with NCoR. If the different TR protein levels caused the correlation between DNE and SMRT, a significant correlation should also be found between DNE and NCoR.
A two-hybrid interference assay is a good, sensitive method for quantitative analysis of RXR binding affinity. Using this method, we found a significant correlation between the RXR-binding activity and the DNE among mTRs. Since heterodimer formation between the TR and the RXR is required for corepressor binding (Zhang et al. 1997), the significant correlation we found seems plausible; an mTR with higher binding affinity to the RXR forms a more stable heterodimer complex with the RXR and, consequently, may recruit SMRT more vigorously. Taken together, the findings suggest the following model for the mechanism of RTH: a mutant TR 
(a) DR4

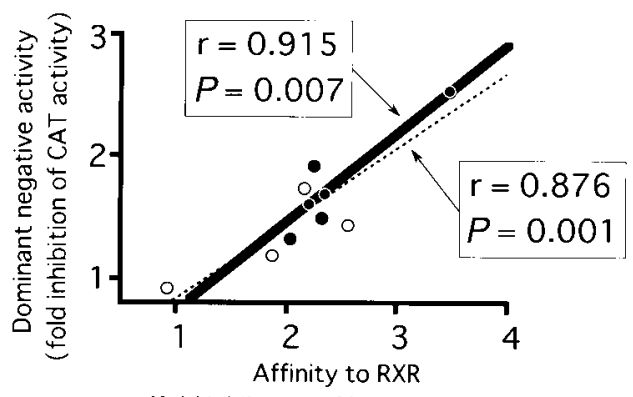

(fold inhibition of luciferase activity)

(b) pal2

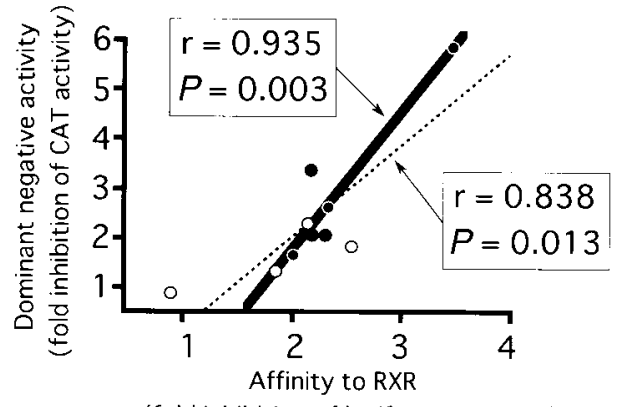

(fold inhibition of luciferase activity)

(c) lap

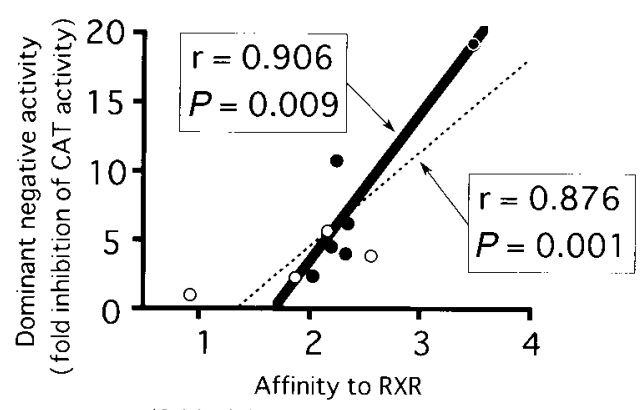

(fold inhibition of luciferase activity)

Figure 8 Correlation between binding avidity of ten mutant TRs for RXR and the dominant negative activity measured on TRE-DR4 (a), TRE-pal2 (b) and TRE-lap (c). The potency of the dominant negative activity was calculated as the ratio between the CAT activities expressed with wt TR and those expressed with mTR (from the data shown in Fig. 2). The binding avidity for RXR was calculated as the ratio between the luciferase activities with expression of $\mathrm{pCMX}$ and those with expression of MTR (data from Fig. 7). Solid lines represent correlations among six natural mTRs ( ); dotted lines represent correlations among all mTRs, including four artificially constructed mTRs $(\bigcirc)$.

heterodimerizes with the RXR and recruits a corepressor (SMRT in our experimental conditions) and can there occupy the target DNA site, the TRE. The receptor complex represses the transcriptional activity of the gene, depending on the binding potency of the mTR for the corepressor. At the same time, the mTR/RXR complex prevents the normal TR/RXR from binding to the TRE and may also titrate out the functional TR and/or some transcriptional factor(s).

\section{Acknowledgements}

The authors are grateful to Drs R M Evans (Howard Hughes Medical Institute, The Salk Institute for Biological Studies, La Jolla, CA, USA), Kazuhiko Umesono (Kyoto University, Kyoto Japan) and Takashi Nagaya (Nagoya University, Nagoya, Japan) for providing the plasmids. This work was supported by a Grant-in-Aid for Scientific Research (to $\mathrm{H} \mathrm{N}$ ) from the Ministry of Education of Japan.

\section{References}

Andoh S, Nakamura H, Sasaki S, Nishiyama K, Kitahara A, Nagasawa S, Mikami T, Natsume H, Genma R \& Yoshimi T 1996 Introducing a point mutation identified in a patient with pituitary resistance to thyroid hormone (Arg 338 to Trp) into other mutant thyroid hormone receptors weakens their dominant negative activities. Journal of Endocrinology 151 287-292.

Baniahmad A, Dressel U \& Renkawitz R 1998 Cell-specific inhibition of retinoic acid receptor-alpha silencing by the AF2/tau c activation domain can be overcome by the corepressor SMRT, but not by N-CoR. Molecular Endocrinology 12 504-512.

Bhat MK, McPhie P, Ting YT, Zhu XG \& Cheng SY 1995 Structure of the carboxy-terminal region of thyroid hormone nuclear receptors and its possible role in hormone-dependent intermolecular interactions. Biochemistry 34 10591-10599.

Chen JD \& Evans RM 1995 A transcriptional co-repressor that interacts with nuclear hormone receptors. Nature 377 454-457.

Clifton Bligh RJ, de Zegher F, Wagner RL, Collingwood TN, Francois I, Van Helvoirt M, Fletterick RJ \& Chatterjee VK 1998 A novel TR beta mutation (R383H) in resistance to thyroid hormone syndrome predominantly impairs corepressor release and negative transcriptional regulation. Molecular Endocrinology 12 609-621.

Collingwood TN, Rajanayagam O, Adams M, Wagner R, Cavailles V, Kalkhoven E, Matthews C, Nystrom E, Stenlof K, Lindstedt G, Tisell L, Fletterick RJ, Parker MG \& Chatterjee VK 1997 A natural transactivation mutation in the thyroid hormone beta receptor: impaired interaction with putative transcriptional mediators. PNAS 94 248-253.

Collingwood TN, Wagner R, Matthews CH, Clifton Bligh RJ, Gurnell M, Rajanayagam O, Agostini M, Fletterick RJ, Beck Peccoz P, Reinhardt W, Binder G, Ranke MB, Hermus A, Hesch RD, Lazarus J, Newrick P, Parfitt V, Raggatt P, de Zegher F \& Chatterjee VK 1998 A role for helix 3 of the TRbeta ligandbinding domain in co-activator recruitment identified by characterization of a third cluster of mutations in resistance to thyroid hormone. EMBO Journal 17 4760-4770.

Crawford PA, Dorn C, Sadovsky Y \& Milbrandt J 1998 Nuclear receptor DAX-1 recruits nuclear receptor corepressor N-CoR to steroidogenic factor 1. Molecular and Cellular Biology 18 2949-2956.

Horlein AJ, Naar AM, Heinzel T, Torchia J, Gloss B, Kurokawa R, Ryan A, Kamei Y, Soderstrom M, Glass CK \& Rosenfeld M 1995 Ligand-independent repression by the thyroid hormone receptor mediated by a nuclear receptor co-repressor. Nature 377 397-404.

Liu Y, Takeshita A, Misiti S, Chin WW \& Yen PM 1998 Lack of coactivator interaction can be a mechanism for dominant negative activity by mutant thyroid hormone receptors. Endocrinology 139 4197-4204

McKenna NJ, Lanz RB \& O’Malley BW 1999 Nuclear receptor coregulators: cellular and molecular biology. Endocrine Reviews $\mathbf{2 0}$ 321-344. 
Meier CA, Parkison C, Chen A, Ashizawa K, Meier HS, Muchmore P, Cheng SY \& Weintraub BD 1993 Interaction of human beta 1 thyroid hormone receptor and its mutants with DNA and retinoid $\mathrm{X}$ receptor beta. T3 response element-dependent dominant negative potency. Journal of Clinical Investigation 92 1986-1993.

Misiti S, Schomburg L, Yen PM \& Chin WW 1998 Expression and hormonal regulation of coactivator and corepressor genes. Endocrinology $1392493-2500$.

Miyoshi Y, Nakamura H, Sasaki S, Tagami T, Misaki T, Konishi J \& Nakao K 1995 Two consecutive nucleotide substitutions resulting in the $\mathrm{T} 3$ receptor beta gene resulting in an 11-amino acid truncation in a patient with generalized resistance to thyroid hormone. Molecular and Cellular Endocrinology 114 9-17

Miyoshi Y, Nakamura H, Tagami T, Sasaki S, Dorin RI, Taniyama M \& Nakao K 1998 Comparison of the functional properties of three different truncatedthyroid hormone receptors identified in subjects with resistance to thyroid hormone. Molecular and Cellular Endocrinology 137 169-176.

Nagaya T \& Jameson JL 1993 Thyroid hormone receptor dimerization is required for dominant negative inhibition by mutations that cause thyroid hormone resistance. Journal of Biological Chemistry 268 15766-15771.

Nagaya T, Kopp P, Kitajima K, Jameson JL \& Seo H 1996 Second zinc finger mutants of thyroid hormone receptor selectively preserve DNA binding and heterodimerization but eliminate transcriptional activation. Biochemical and Biophysical Research Communications 222 $524-530$.

Nagaya T, Fujieda M \& Seo H 1998 Requirement of corepressor binding of thyroid hormone receptor mutants for dominant negative inhibition. Biochemical and Biophysical Research Communications 247 $620-623$.

Nishiyama K, Andoh S, Kitahara A, Natsume H, Mikami T, Genma R \& Nakamura H 1998 Difference in dominant negative activities between mutant thyroid hormone receptors $\alpha 1$ and $\beta 1$ with an identical truncation in the extreme carboxyl-terminal tau 4 domain. Molecular and Cellular Endocrinology 138 95-104.

Nishiyama K, Matsushita A, Natsume H, Mikami T, Genma R, Sasaki S \& Nakamura H 2000 Differences between the silencing-related properties of the extreme carboxyl-terminal regions of thyroid hormone receptor $\alpha 1$ and $\beta 1$. Journal of Endocrinology 167 221-229.

Nordeen SK, Green PP III \& Fowlkes DM 1987 A rapid, sensitive, and inexpensive assay for chloramphenicol acetyltransferase. DNA 6 173-178.

Refetoff S, DeWind LT \& DeGroot LJ 1967 Familial syndrome combining deaf-mutism, stippled epiphyses, goiter and abnormally high PBI: possible target organ refractoriness to thyroid hormone. Journal of Clinical Endocrinology 27 179-294.

Safer JD, Cohen RN, Hollenberg AN \& Wondisford FE 1998 Defective release of corepressor by hinge mutants of the thyroid hormone receptor found in patients with resistance to thyroid hormone. Journal of Biological Chemistry 273 30175-30182.
Sakurai A, Takeda K, Ain K, Ceccarelli P, Nakai A, Seino S, Bell GI, Refetoff S \& DeGroot LJ 1989 Generalized resistance to thyroid hormone associated with a mutation in the ligand-binding domain of the human thyroid hormone receptor beta. PNAS $\mathbf{8 6}$ 8977-8981.

Samuels HH, Stanley F \& Casanova J 1979 Depletion of L-3,5,3'triiodothyronine and L-thyroxine in euthyroid calf serum for use in cell culture studies of the action of thyroid hormone. Endocrinology $10580-85$.

Sasaki S, Nakamura H, Tagami T, Miyoshi Y, Tanaka K \& Imura H 1992 A point mutation of the T3 receptor beta 1 gene in a kindred of generalized resistance to thyroid hormone. Molecular and Cellular Endocrinology 84 159-166.

Sasaki S, Nakamura H, Tagami T, Miyoshi Y, Nogimori T, Mitsuma T \& Imura H 1993 Pituitary resistance to thyroid hormone associated with a base mutation in the hormone-binding domain of the human 3,5,3'-triiodothyronine receptor-beta. Journal of Clinical Endocrinology and Metabolism 76 1254-1258.

Tagami T \& Jameson JL 1998 Nuclear corepressors enhance the dominant negative activity of mutant receptors that cause resistance to thyroid hormone. Endocrinology 139 640-650.

Tagami T, Gu WX, Peairs PT, West BL \& Jameson JL 1998 A novel natural mutation in the thyroid hormone receptor defines a dual functional domain that exchanges nuclear receptor corepressors and coactivators. MolecularEndocrinology 12 1888-1902

Takeda K, Sakurai A, DeGroot LJ \& Refetoff S 1992 Recessive inheritance of thyroid hormone resistance caused by complete deletion of the protein-coding region of the thyroid hormone receptor-beta gene. Journal of Clinical Endocrinology and Metabolism 74 49-55.

Yen PM \& Chin WW 1994 Molecular mechanisms of dominant negative activity by nuclear hormone receptors. Molecular Endocrinology 8 1450-1454.

Yoh SM, Chatterjee VK \& Privalsky ML 1997 Thyroid hormone resistance syndrome manifests as an aberrant interaction between mutant T3 receptors and transcriptional corepressors. Molecular Endocrinology 11 470-480.

Zamir I, Harding HP, Atkins GB, Horlein A, Glass CK, Rosenfeld MG \& Lazar MA 1996 A nuclear hormone receptor corepressor mediates transcriptional silencing by receptors with distinct repression domains. Molecular and Cellular Biology 16 5458-5465.

Zhang J, Zamir I \& Lazar MA 1997 Differential recognition of liganded and unliganded thyroid hormone receptor by retinoid $\mathrm{X}$ receptor regulates transcriptional repression. Molecular and Cellular Biology 17 6887-6897

Received 2 June 2000

Revised manuscript received 28 July 2000 Accepted 22 August 2000 